\title{
TRAINING OF DRIVING-RELATED ATTENTIONAL PERFORMANCE AFTER STROKE USING A DRIVING SIMULATOR
}

\author{
Emmanuel Abiodun Akinwuntan \\ School of Allied Health Sciences \\ Medical College of Georgia \\ Augusta, GA USA \\ E-mail: aakinwuntan@mcg.edu
}

Willy De Weerdt, Hilde Feys, Geert Verheyden, F. De Vooght, Hannes Devos

Department of Rehabilitation Sciences

Faculty of Kinesiology and Rehabilitation Sciences

Katholieke Universiteit Leuven, Belgium

Guido Baten

CARA, Belgian Road Safety Institute

Brussels, Belgium.

Carlotte Kiekens

Department of Physical Medicine and Rehabilitation

University Hospital Pellenberg, Belgium

\begin{abstract}
Summary: The objective of this study was to determine the effect of simulatorbased driving training on attentional performance after stroke. A further analysis of data was conducted from a randomized controlled trial in which the effect of simulator training and cognitive paper and pencil training to improve driving were compared. Performance in divided attention tasks before, during and after 15 hours of simulator-based training of general driving skills in 33 experimental participants were evaluated. Performance in divided attention tasks was assessed during navigation of a 5-km scenario with the divided attention tasks as the only event to respond to and another $13.5-\mathrm{km}$ scenario that contained a good mixture of regular day to day traffic situations. There were significant improvements in mean response time to the divided attention tasks and time to complete the $5-\mathrm{km}$ scenario. Significant decrease in mean response time, number of missed responses, collisions, pedestrians hit, total faults and run time and increase in number of correct responses were found in the 13.5-km scenario. Further analyses showed most improvements in the simulator assessments occurred between preand mid-training. Simulator-based training of driving skills positively impacted attentional performance. Findings in this study suggest that 10 hours of simulatorbased driving training after stroke is sufficient to realize meaningful benefits.
\end{abstract}

\section{INTRODUCTION}

Different types of attention failure are involved in motor vehicle accidents with visual attention deficits (divided attention, sustained attention and selective attention) being the most common. A meta-analysis of 30 studies by Park \& Ingles in 2001 evaluated the efficacy of retraining attention after acquired brain injuries revealed that improvement due to training did not tend to generalize or transfer to tasks that differ considerably from those used in training. Currently available driving simulators, however, have the potential to be used to train divided attention in a 
driving context (Akinwuntan et al., 2005), though the efficacy of such training, especially after stroke, has not been established. The effectiveness of driving training after stroke using a driving simulator was reported in a recent randomized controlled study that included 83 first-ever subacute stroke survivors (Akinwuntan et al., 2005). Participants were randomly allocated to either an experimental group (42) that received 15 hours of structured simulator-based driving training or a control group (41) that received 15 hours of structured driving-related paper and pencilbased cognitive training. Significantly more experimental subjects passed an official driving assessment administered about 3 months after completion of the 15 hour simulator-based training and were legally allowed to resume driving. Part of the training received by the experimental participants included simulator-based training of attentional performance. In this study, the data obtained from the randomized controlled trial are further analyzed to determine the effect of simulator-based driving training on attentional performance after stroke. We also investigated if any of the general descriptive characteristics of participants, including side of lesion, would predict improvement in important attentional performance parameters.

\section{METHODS}

Participants' general descriptors such as age, sex, level of education, side of lesion, driving history, overall disability and mental state were documented before commencement of training. Data of performances in the tests of kinetic vision, perimetry, Useful Field of View (UFOV) and on-road driving recorded during the pre-training evaluation were also retrieved. A kinetic vision test was used to evaluate the ability to recognize objects in motion. The test involved presentation of 9 chevrons that moved horizontally from left to right at 3 different speeds while participants identified whether the chevrons pointed left or right. A maximum score of 3 was given for 8 or 9 correct responses and 0 for 8 or 9 wrong answers. During the perimetry test, the participants had to fix their eyes on a central point. A computer-driven program flashed small lights at different locations of the visual field and the number of misses was registered. No misses was documented as normal. Two or more misses in one quadrant of one or both eyes' visual fields were documented as quadrant-anopia. Three or more misses in one half of one or both eyes' visual fields were documented as hemi-anopia. The UFOV test measured the degree of reduction in the useful field of view by evaluating the speed of processing, divided and selective attention of performance using visual discrimination and localization tasks (Ball et al., 1990). The on-road test was performed in an adapted car. It consisted of a 13-item checklist that contained 49 sub-items (Akinwuntan et al., 2005). Each sub-item was scored on a 4-point scale of 1 for worst performance and 4 for best performance, which resulted in minimum of 49 and maximum of 196 obtainable points. Based on performance in the on-road test, participants were classified by a driving expert as 1) "fit to drive," 2) "temporarily unfit to drive," or 3) "unfit to drive."

In addition to regular hospital rehabilitation, participants in both the experimental and control groups received 15 hours of additional training spread over 5 weeks at 1 hour per day, three days a week. Those in the experimental group received simulator-based training of general driving skills, which included divided attention skills. The divided attention task involved locating two red diamond shapes displayed at the middle left and right edges of the screen. During driving, the diamonds changed one at a time randomly to either a triangle or horn sign, and returned to the diamond shape after 10 seconds or immediately upon action being taken. When the right or left 
diamond shape changed to a triangle, the experimental participants were instructed to signal a right or left turn as fast as possible. When the right or left diamond changed to a horn shape, participants were instructed to blare the horn. To get acquainted with the driving simulation, use of adaptive aids such as a steering spinner knob and left-sided gas pedal when needed and the divided attention tasks, a 3-km scenario designed to enable gradual familiarization was used. On average, it took participants 2 to 3 hours for full familiarization, after which driving performance was assessed in the simulator using a $13.5-\mathrm{km}$ scenario of regular day to day traffic. The $13.5-\mathrm{km}$ scenario also contained divided attention tasks. Number of collisions, pedestrians hit, speed exceedances, traffic light faults, total faults and time to complete the 13.5-km scenario were evaluated during this assessment, along with performance in divided attention tasks. A second 5$\mathrm{km}$ long scenario that contained only divided attention tasks was also presented to specifically assess performance in the task. During both assessments, the mean response time to 20 changes in the diamond shapes, numbers of correct and missed responses were automatically documented by the simulator computer. Performance during the first navigation of the $13.5 \mathrm{~km}$ and $5 \mathrm{~km}$ scenarios were used as pre-training evaluation. For training general driving and divided attention skills, different, short, 5-km scenarios that were not similar to the assessment scenarios but required similar driving skills were used. The $13.5-\mathrm{km}$ and $5-\mathrm{km}$ assessment scenarios were again presented after 10 hours and 15 hours of simulator-based driving to evaluate the mid- and post-training performances. Only the data of 33 of the 37 experimental participants who completed the 15 hours of training with complete data sets of performance in the divided attention tasks in both the 13.5- and 5-km scenarios were used in this study.

Participants (36) in the control group received structured driving-related paper and pencil-based cognitive training for 15 hours, as well performing cognitive tasks such as route finding on a paper or road map, memory training with numbers, and forming different patterns using tiles. Recognition of road and traffic signs was also trained using 40 cards with pictures of different traffic situations.

\section{Data Analysis}

Pre- through mid- to post-training improvements in the divided attention tasks in the experimental group in the $13.5-\mathrm{km}$ and 5-km scenarios were determined using Kruskal-Wallis tests. Wilcoxon signed rank test was used to evaluate pre- to mid-training and mid- to posttraining improvements.

Univariate and multiple regression analyses were performed, respectively, to investigate if any or a combination of the general descriptive characteristics of participants in the experimental group including age, sex, level of education, side of lesion, driving history, overall disability and mental state or visual, cognitive-perceptual and on-road driving abilities would predict improvement in mean response time and number of correct responses in the divided attention tasks of the 13.5-, 5- and 5-km scenarios.

The general descriptive characteristics of participants in the experimental group were compared to those of the controls using Chi square for nominal data, Mann-Whitney U test for ordinal data or not normally distributed ratio variables, and unpaired $t$ tests for normally distributed ratio variables. The differences between the groups at pre- and post-training assessments, as well as improvements from pre- to post-training, in the tests of kinetic vision, perimetry, UFOV and on- 
road performance were evaluated using Mann-Whitney U tests. Within-group improvements in the three tests in each group were determined by comparing outcome of post- with pre-training driving assessments using Wilcoxon signed rank tests. The decisions of driving fitness (in three classes) based on the on-road test were compared between groups pre- and post-training and for improvements from pre- to post-training using Chi square tests.

Normal distribution of interval/ratio variables in all cases was checked using the KolmogorovSmirnov test. For significance testing, $\mathrm{p} \leq 0.05$ was considered significant. All statistical procedures were performed with the SAS System (Freund \& Litell, 1991).

\section{RESULTS}

Mean response time to the divided attention tasks decreased significantly $(\mathrm{p}=.0008)$ from 1.6 seconds pre-training to 1.3 seconds post-training. This finding was predominantly due to the decrease in mean response time that occurred after 10 hours of training in the driving simulator, i.e., between pre- and mid-training assessments, as seen in Table 1. A significant decrease $(p=.0405)$ in time required to complete the $5-\mathrm{km}$ scenario was also found but only after 15 hours of training. The number of correct and missed responses was not affected.

Table 1. Performance in the divided attention tasks at pre-, mid- and post-training and comparison between pre- and mid-training, mid- and post-training and from pre- to posttraining $(\mathbf{n}=33)$

\begin{tabular}{|c|c|c|c|c|c|c|}
\hline Variables & Pre-training & Mid-training & Post-training & Pre-Mid* & Mid-Post* & Pre-Post $* *$ \\
\hline 5 km Scenario & \multicolumn{3}{|c|}{ Median(IQR) } & \multicolumn{3}{|c|}{$p$-values } \\
\hline Mean resp. time, sec & $1.6(1.3-2.6)$ & $1.4(1.1-1.7)$ & $1.3(1.0-1.8)$ & .0001 & .3828 & .0008 \\
\hline Correct response, $\mathrm{n}$ & $15(9-18)$ & $16(9-18)$ & $16(10-18)$ & .6469 & .9640 & .6518 \\
\hline Missed response, $\mathrm{n}$ & $0(0-0)$ & $0(0-0)$ & $0(0-0)$ & .2656 & 1.000 & .2656 \\
\hline Excessive speed, $\mathrm{n}$ & $0(0-0)$ & $0(0-0)$ & $0(0-0)$ & .1382 & 1.000 & .0630 \\
\hline Run time, sec & $362(286-429)$ & $338(294-379)$ & $313(276-350)$ & .3670 & 1.000 & .0405 \\
\hline \multicolumn{7}{|l|}{$13.5 \mathrm{~km}$ Scenario } \\
\hline Mean resp. time, sec & $4.8(3.7-7.7)$ & $2.8(2.0-4.8)$ & $2.8(1.6-4.1)$ & $<.0001$ & .0047 & $<.0001$ \\
\hline Correct response, $\mathrm{n}$ & $7(2-11)$ & $11(6-13)$ & $13(8-13)$ & $<.0001$ & .4667 & $<.0001$ \\
\hline Missed response, $\mathrm{n}$ & $6(3-11)$ & $2(0-5)$ & $1(0-4)$ & $<.0001$ & .1715 & $<.0001$ \\
\hline Excessive speed, $\mathrm{n}$ & $0(0-0)$ & $0(0-0)$ & $0(0-1)$ & .3564 & .0840 & .6333 \\
\hline Run-time, sec & $1298(1121-1401)$ & $1190(1098-1314)$ & $1153(1057-1246)$ & .0002 & .1271 & $<.0001$ \\
\hline Collisions, $\mathrm{n}$ & $1(0-3)$ & $0(0-0)$ & $0(0-0)$ & .0046 & 1.000 & .0002 \\
\hline Pedestrians hit, $\mathrm{n}$ & $2(1-2)$ & $0(0-1)$ & $0(0-1)$ & $<.0001$ & .1447 & $<.0001$ \\
\hline Traffic light faults, $\mathrm{n}$ & $0(0-1)$ & $0(0-0)$ & $0(0-0)$ & .5078 & .5313 & .1094 \\
\hline Total faults, $\mathrm{n}$ & $7(6-9)$ & $2(0-3)$ & $0(0-3)$ & $<.0001$ & .0886 & $<.0001$ \\
\hline
\end{tabular}

$\mathrm{IQR}=$ Interquartile range $\left(\mathrm{Q}_{1}-\mathrm{Q}_{3}\right), *=$ Wilcoxon Signed Rank test, $* *=$ Kruskal-Wallis test 
Performance in the 13.5-km scenario revealed significant decreases in mean response time $(\mathrm{p}=<.0001)$ and number of missed responses $(\mathrm{p}=<.0001)$ and increase in number of correct responses $(\mathrm{p}=<.0001)$ in the divided attention tasks that was embedded in the program.

Significant decrements in number of collisions $(p=.0002)$, number of pedestrians hit $(p=<.0001)$, total faults $(\mathrm{p}=<.0001)$ and time required to complete the program $(\mathrm{p}=<.0001)$ were observed as well. Further analyses revealed that most improvement in the parameters assessed in the $13.5 \mathrm{~km}$ scenario occurred from pre- to mid-training. Only mean response time showed continued significant improvement from mid- to post-training. Number of excessive speed violations in both the 5-km and 13.5-km scenarios showed no significant decrease from pre- through mid- to post-training. The number of traffic light faults was very low during all three assessment periods.

Outcome of the univariate regression analyses did not reveal any descriptive characteristics of participants in the experimental group, including side of lesion or visual, cognitive-perceptual and on-road driving abilities as a predictor of the significant improvement in mean response time to the divided attention tasks in the 5-km scenario. Multiple regression analyses also did not reveal any combination of these same variables as the best to predict the improvement in mean response time. In the $13.5-\mathrm{km}$ scenario, both $\operatorname{sex}(p=.02)$ and side of lesion $(p=.04)$ were single predictors of the significant decrement in mean response time from pre-to post-training, and multiple regression analysis showed a combination of both tests as the best predictor $\left(\mathrm{R}^{2}=0.25\right)$. Side of lesion again $(\mathrm{p}=.05)$ and level of education $(\mathrm{p}=.01)$ were the single predictors of the significant increase in the number of correct responses from pre-to post-training. Female experimental participants with left sided lesion showed the most decrement in the mean response time. Multiple regression analysis showed a combination of both parameters and average distance driven annually pre-stroke as the best model $\left(\mathrm{R}^{2}=0.31\right)$ to predict the significant increase in the number of correct responses. Data exploration revealed that well educated stroke survivors with left sided lesions who covered relatively more mileage annually before stroke onset showed the best improvement in responding correctly to the divided attention tasks.

No differences in the general descriptive characteristics were found at pre-training between experimental and control groups. The same was observed for performances in the kinetic vision, perimetry, UFOV and on-road tests, as well as in the three-class decision of driving fitness. Performances in the on-road test $(\mathrm{p}=.02)$ and in the three-class decision of fitness-to-drive $(p=.03)$ had, however, become significant post training. There were significant within-group improvements in both groups in all the tests from pre- to post-training with the exception of performance in the perimetry tests $(\mathrm{p}=0.04)$ in the experimental group. When the improvements were compared between groups, only the fitness-to-drive decisions made based on performance in the on-road test were significantly different and in favor of the experimental group.

\section{DISCUSSION}

In the 5-km scenario, there was significant decrement in the mean time required to respond to the divided attention tasks, yet there was no increment in the number of correct responses. These findings suggest an improvement in simple rather than choice reaction time, as was also reported by Klavora et al. in 1995. The effect of driving skills training on performance in the divided attention tasks was more evident in the $13.5-\mathrm{km}$ scenario, with significant increment in number of correct responses and decrement in mean response time and number of missed responses in 
the divided attention tasks. It is possible that the $13.5-\mathrm{km}$ scenario with regular day to day traffic was more realistic for the experimental participants and so they gave their best performance. It is also possible that the 5-km scenario, with only the divided attention tasks and a pre-training average score of 15 out of 20 , was too simple to detect impairments pre-training and consequently any improvements from pre- to post-training. However, performances in the divided attention tasks during the pre- through mid- to post-training assessments were worse in the $13.5-\mathrm{km}$ scenario in comparison to similar performances in the 5-km scenario. The more detailed complex traffic events presented in the $13.5-\mathrm{km}$ scenario definitely required higher mental workload, which led to poorer detection abilities, a finding that had been reported in another study (Recarte \& Nunes, 2003).

Though the relationship between better improvement in mean response time and sex is unclear, left sided lesion stroke survivors were reported to demonstrate less cognitive and visual problems that affect driving when compared to those with right sided lesions (Marshall et al., 1997). High motivation to return to pre-stroke routine, especially in less impaired and well educated stroke survivors, was associated with benefit from the simulator-based driving training in the original randomized controlled trial (Akinwuntan et al., 2005), which led to this present study. The same reason may explain the fact that well educated stroke survivors who had left sided lesions and are habitual drivers pre-stroke showed the best improvement in responding correctly to the divided attention tasks in the $13.5-\mathrm{km}$ scenario.

Improvements from pre- to post-training in tests of kinetic vision, perimetry, UFOV, and on-road driving performance from pre to post-training were not significantly different between the groups, probably because both groups received driving-related training. However, the significant difference in improvement in the fitness-to-drive decisions in favor of the experimental group indicated that the simulator-based driving training resulted in better overall driving performance. As was suggested in the original randomized controlled trial, some crucial factors in the simulator training, which enabled contextual training, may have led to better positive changes in overall driving fitness.

\section{CONCLUSION}

Simulator-based training of driving skills positively impacted attentional performance. Findings in this study suggest that 10 hours of simulator-based driving training after stroke is sufficient to realize meaningful benefits. Both simulator- and paper-based trainings positively impacted performance in tests of kinetic vision, perimetry, UFOV and on-road driving. However, simulator-based driving training resulted in better improvement in overall fitness-to-drive after stroke.

\section{ACKNOWLEDGMENTS}

The primary author expresses profound appreciation to his Belgian colleagues, particularly those at the CARA section of the Belgian Institute for Road Safety, Brussels, Belgium, for support during the conduction of this study. The primary author also acknowledges the Department of Physical Therapy at the Medical College of Georgia, Augusta, GA, for funding support to attend the conference. 


\section{REFERENCES}

Akinwuntan A.E., De Weerdt W., Feys H., Baten G., Arno P., Kiekens C. (2005). The validity of a road test after stroke. Arch Phys Med Rehabil, 86, 421-426.

Akinwuntan A.E., De Weerdt W., Feys H., Baten G., Arno P., Strypstein E., Kiekens C. (2005). Effect of simulator training on the driving ability of stroke patients: a randomized controlled trial (RCT). Neurology, 65, 843-850.

Ball K.B., Roenker D.L., Bruni J.R. (1990). Developmental changes in attention and visual search throughout adulthood. In: Enns J, ed. Advances in psychology. Amsterdam, the Netherlands: Nort-Holland - Elsevier Science Publishers, 489-508.

Freund R.J., Litell R.C. (1991). SAS system for regression. 2nd ed. Cary, NC: SAS Institute.

Klavora P., Gaskovski P., Martin K., Forsyth R.D., Heslegrave R.J., Young M., Quinn R.P. (1995). The effects of dynavision rehabilitation on behind-the-wheel driving ability and selected psychomotor abilities of persons after stroke. Am J Occup Ther, 49, 534-542.

Park N.W., Ingles J.L. (2001). Effectiveness of attention rehabilitation after an acquired brain injury: a meta-analysis. APA Journals, 15, 199-210.

Marshall S.C., Grinnell D., Heisel B., Newall A., Hunt L. (1997). Attentional deficits in stroke patients: a visual dual task experiment. Arch Phys Med Rehabil, 78, 7-12.

Recarte M.A., Nunes L.M. (2003). Mental workload while driving: effects on visual search, discrimination and decision making. J Exp Psychol Appl, 9, 119-137. 